\title{
Are Wild Wolves Southpaws? Including Potential Conservation Implications
}

\author{
Shannon Michelle Barber-Meyer \\ U. S. Geological Survey, Northern Prairie Wildlife Research Center, $871137^{\text {th }}$ St. SE, Jamestown, North Dakota, 58401 United
} States

Email: sbarber-meyer@usgs.gov

Citation - Barber-Meyer, S. M. (2022). Are wild wolves southpaws? Including potential conservation implications. Animal Behavior and Cognition, 9(1), 72-79. https://doi.org/10.26451/abc.09.01.06.2022

\begin{abstract}
Lateralization, or a left-right bias in behavior (e.g., handedness), was originally thought to exclusively exist in humans, but is now known to be widespread. Lateralization can exist at the individual or group level. In dogs (Canis lupus familiaris), tests of paw preference have produced inconsistent results. Because wolves (C. l.) differ genetically, morphologically, and behaviorally from dogs, I was interested in assessing them for lateralization. I examined lateralization (right versus left) of the foot captured (a step test analog) of wild wolves $(n=93)$ trapped for radiocollaring purposes in the Superior National Forest, Minnesota from $2011-2017$ and 2019. No support was found for lateralization, and sex and age class were not significant predictors of which foot was captured. Because many mammals demonstrate lateralization, and because population-level lateralization is thought to convey increased social cohesion, it is surprising that wild wolves did not demonstrate population level lateralization. This step test analog may not have been an appropriate measure (as lateralization is task dependent) and / or wolf lateralization may exist at the individual level, but not the population level. Future work on wolf lateralization at both the individual and population levels examining pawedness via multiple tasks while accounting for potential confounding factors (such as different rearing conditions and methods) could provide clarification. Examining potential trade-offs between the costs and benefits of lateralization that these highly social animals may incur would be very interesting in terms of evolution and in comparison with dogs. Furthermore, because lateralization has been connected to emotional functioning and animal welfare, baseline lateralization data from wild wolves may inform captive wolf management and conservation, including the captive breeding programs for endangered Mexican wolves (C. l. baileyi) and red wolves (C. rufus) and other programs (e.g., educational facilities).
\end{abstract}

Keywords - Canis lupus, Foot-hold trapping, Gray wolves, Handedness, Laterality, Paw preference, Pawedness

Lateralization, or a left-right bias in behavior (e.g., handedness), was originally thought to exclusively exist in humans (Halpern et al., 2005). Now, it is understood to be widespread and possibly even a universal characteristic of vertebrates (Reddon \& Hurd, 2009). There are even reports of lateralized behavior among invertebrates (see Halpern et al., 2005 for review). Some examples where lateralization has been documented include visual scanning for prey in common wall lizards (Podarcis muralis) (Bonati et al., 2008), motor responses in lab rats (Rattus norvegicus) related to whisker sensations (Aggestam \& Cahusac, 2007), jaw movements in ruminating sheep (Ovis aries) (Versace et al., 2007), aggressive responses in domestic chicks (Gallus gallus domesticus) (Vallortigara et al., 2001) and sheepdogs (Canis lupus familiaris) (Siniscalchi et al., 2019), agnostic responses and vigilance in feral horses (Equus caballus) (Austin \& Rogers, 2012) and Przewalski horses (E. przewalskii) (Austin \& Rogers, 2014), and turns in shoaling fish (multiple species) (Bisazza et al., 2000). 
Lateralization can exist at the individual or group (population or species) level (Vallortigara \& Rogers, 2005), with the strength (or degree) of lateralization determined by consistency. Strong laterality in an individual is related to the enhanced ability to process multiple stimuli simultaneously (Vallortigara \& Rogers, 2005). The left cerebral hemisphere is thought to attend to "considered responses," including tasks such as prey discrimination and foraging whereas the right cerebral hemisphere is thought to attend to "rapid, species-typical responses," such as predator detection and avoidance (see Vallortigara \& Rogers, 2005 for review). For example, lateralized individual domestic chicks were better at detecting predators while also foraging (Rogers et al., 2004). Both cerebral hemispheres are also involved in emotional processing, with emotional hemispheric dominance (laterality) based on whether it is a negatively or positively connotated emotion (Leliveld et al., 2013; Quaranta et al. 2007) or whether it is perceived as a stressful stimulus (Rogers, 2010; Siniscalchi et al., 2021). For example, in domestic dogs positive stimuli produced a higher amplitude of tail wagging to the right side, whereas a higher amplitude of tail wagging to the left side was observed in response stimuli that resulted in withdrawal behavior (Quaranta et al. 2007). Laterality has also been related to superior task ability, such as in domestic cats (Felis catus) that demonstrated more accurate and faster reactions (Fabre-Thorpe et al., 1993) and in wild chimpanzees (Pan troglodytes schweinfurthii) that were more successful at foraging for termites (Macrotermes spp.) when using the same hand consistently (McGrew \& Marchant, 1999). Lateralization has also been related to behaviors such as aggression, stress response, boldness, fear, and coping mechanisms, as well as animal welfare and emotional functioning (Austin \& Rogers, 2012; Branson \& Rogers, 2006; Leliveld et al., 2013; Neveu \& Merlot, 2003; Reddon \& Hurd, 2009; Rogers, 2010; Sinisclachi et al., 2019, 2021; Wells, 2021).

While lateralization at the individual level is related to enhanced neural activity and presents advantages in many situations, strong lateralization in one direction at the population level (more than 50\% of the group show the same lateral bias in natural behavior, Vallortigara \& Rogers, 2005) can be disadvantageous (Halpern et al., 2005; Reddon \& Hurd, 2009). For example, a prey population strongly lateralized to detect predators on the same side would be vulnerable from attack on the other side, whereas predators strongly lateralized to attack prey on the same side would be only half as likely to capture prey on average (Halpern et al., 2005; Vallortigara \& Rogers, 2005). Given such disadvantages, why does population-level lateralization exist? It has been proposed that it is a mechanism to coordinate social behavior and promote social stability through predictable individual lateralized behavior (Rogers, 1989; Vallortigara \& Rogers, 2005). For example, fish that shoal in the open ocean show population level lateralized turning behavior when reaching an object, whereas non-shoaling fish are lateralized only at the individual level (see Halpern et al., 2005 for review). Similarly, fish (Brachyrhaphis episcopi) from highpredation areas also exhibited stronger lateralization (Brown et al., 2007).

Such population level lateralization may persist as an evolutionarily stable strategy (Maynard Smith, 1982) where the cost / benefit of an individual's strategy depends in a frequency-dependent manner on the strategies of others in the group. The costs to an individual for population-level lateralization (e.g., increased predictability when being attacked or when foraging) are assessed relative to the benefits (e.g., coordinated social behavior, increased neural capacity to the individual), given the strength and direction of lateralization of other individuals in the group (Vallortigara \& Rogers, 2005).

Lateralization has been shown to have a heritable component (Brown et al, 2007). Nevertheless, conditions during embryonic development (e.g., hormones, stimulation with light, rotation of the embryo, body position) can influence the direction and presence of lateralization (see Halpern et al., 2005 for review). Environmental conditions can even reverse or abolish lateralization (Halpern et al., 2005). Thus, the relationship between genetics and epigenetics are both important in the realization of the degree and direction of lateralization (Halpern et al., 2005). Sex has also been correlated with lateralization (e.g., domestic dogs and cats; see Wells, 2021 for review). Lateralized male convict cichlid fish (Archocentrus nigrofasciatus) were more aggressive than weakly lateralized males, but lateralized female fish were less aggressive than weakly lateralized females (Reddon \& Hurd, 2008). The influence of embryonic hormones on lateralization is likely a cause of some of the observed sex-based lateralization differences (Halpern et al., 2005). In addition to sex, age class (e.g., Austin \& Rogers, 2014) has also been related to lateralization. Further, the role of socio-cultural factors has been investigated as it relates to lateralization, as in the 
proportion of right-to-left handed individuals (2:1 in non-human primates versus 8 or 9:1 in humans) (see Halpern et al., 2005 for review).

Assessing lateralization can be difficult because lateralization can be task-dependent and may depend also on the complexity or novelty of the task (Batt et al., 2008; Halpern et al., 2005; Poyser et al., 2006; Tomkins et al., 2010). Thus, using different methods can yield conflicting results (see Halpern et al., 2005; Poyser et al., 2006; Tomkins et al., 2010), making comparisons across studies challenging. In dogs, lateralization tests have involved foraging, grooming and locomotory behaviors (Poyser et al., 2006; Tomkins et al., 2010; Wells, 2003, 2021). Lateralized paw preference has been evaluated in first stepping, removing tape or a blanket, paw shaking, and handling a ball or food / chew toy (Batt et al., 2008; Berta, 2011; Drew 2015; Poyser et al., 2006; Tomkins et al., 2010; Wells et al., 2016). Results of such tests have been mixed. Dogs were initially determined to exhibit right paw preference at the population level (Tan, 1987), but they were tested on only one task that required them to remove adhesive plaster from over their eyes. Wells (2003) conducted later work using three tasks that found sex-biased differences in the direction (but not the strength) of the lateralization. Subsequent work by Wells et al. (2016) found that dogs may preferentially use their non-dominant paw for manipulation in a task while using their dominant paw for postural stabilization. Another study found that male dogs were biased to their left paw, whereas females were biased to their right, and the authors related this to immune function modulated by brain asymmetry (Quaranta et al., 2004). However, a later study found that sex-biased lateralization is labile and may relate to task novelty in that male dogs were only biased to the left paw initially (first encounter) in a ball test, one of three tests conducted (Poyser et al., 2006). Because it is suspected that the right hemisphere of the brain is used to respond to novelty (see Poyser et al., 2006), the researchers postulated that the observed differences in initial lateralization between the sexes may be explained by differences between male and female brains and how their response to novelty differs regardless of whether the task requires a lateralized response (Poyser et al., 2006). They found no such lateralization with respect to first paw use in their chew test, however (Poyser et al., 2006). Whether sex was related to lateralization in dogs could have also differed among studies depending on whether the subjects were sexually intact or neutered (Wells, 2003). Although it has been proposed that young animals are not as lateralized as adults (MacNeilage et al., 1987), age effects have not been rigorously evaluated in dogs (Wells, 2003), but have been related to lateralization in one test (Tomkins et al., 2010). An alternate interpretation of conflicting test results that was suggested by a reviewer is the possibility that a paucity of replication, lack of controls, sometimes inadequate sample sizes, and multiple statistical tests without clear a priori hypotheses can result in statistical noise rather than confirmation of biological significance (e.g., Yoccoz, 1991).

While dogs have been studied for decades, very little is known about gray wolves $(C . l$.$) and$ lateralization. Although wolves and dogs are closely related, they are different species and the process of domestication has altered dog genetics, morphology, and behaviors (Björnerfeldt, 2007). Because genetics and different life experiences, including rearing conditions, may affect lateralization direction and degree (Brown et al., 2007), wolves and dogs could differ in lateralization. Thus, although results from lateralization tests of domestic dogs exist, research on wolves, especially wild wolves, remains warranted. A previous study (Drew, 2015) of captive wolves, including three red wolves (C. rufus) and seven gray wolves of three subspecies, used a chew/handling test and a step test to conclude that the presence of lateralization varied by task and method of calculation. Not all wolves participated in both tasks (step test $=5$ wolves, chew/handling test $=9$ wolves) and sex and age effects were not evaluated. Total paw touches by all wolves in the step test was biased left $(70 \%$ or 119 touches with left, $30 \%$ or 51 touches with right paw) - but at the individual level, two wolves were characterized as left-biased, two were right-biased, and one was ambidextrous (Drew, 2015). No lateralization was found in the chew/handling test (Drew, 2015). Varying methods for performing these tests were used at the three different captive wolf locations (Drew, 2015), but data from all locations were pooled in the analysis. Notably, different laboratory / rearing conditions (Brown et al., 2007), can result in different lateralization degree and direction.

Wild wolves could further be differentiated from captive wolves in lateralization because animals in regular contact with humans may exhibit learned behaviors rather than behaviors based on neurobiological lateralization (Brown et al., 2007; Poyser et al., 2006; Wells, 2003). Thus, I assessed wild 
wolf lateralization at the population level via the foot captured in traps for radiocollaring purposes. Because so many mammals demonstrate lateralization (Reddon \& Hurd, 2009) and because population-level lateralization (of which, handedness is an example) is thought to convey increased social cohesion (Vallortigara \& Rogers, 2005), I hypothesized that wolves, a highly social species (Lampe et al., 2017; Mech et al., 2015; Range \& Virányi, 2015), would similarly exhibit this characteristic through paw preference at the population level.

Method

\section{Ethics Statement}

The guidelines of the American Society of Mammalogists for use of wild mammals in research (Sikes et al., 2016) were followed and this work was conducted under USFWS permits PRT831774 and TE3886A-0 and the approval of the U.S. Geological Survey, Northern Prairie Wildlife Research Center Animal Care and Use Committee. For additional details on trapping, capture, and processing see BarberMeyer and Mech (2014).

\section{Study Area}

The study area included $2,060-\mathrm{km}^{2}$ in the Superior National Forest, Minnesota, USA $\left(48^{\circ} \mathrm{N}, 92^{\circ} \mathrm{W}\right.$; see Nelson \& Mech, 1981 for a detailed description). Temperatures rarely exceeded $35^{\circ} \mathrm{C}$. Elevations ranged from $325 \mathrm{~m}$ to $700 \mathrm{~m}$ above sea level and included swamps, uneven upland, and rocky ridges (Mech, 2009). Vegetation was predominately conifers (e.g., jack pine [Pinus banksiana], white pine [P. strobus], red pine $[P$. resinosa], black spruce [Picea mariana], white spruce $[P$. glauca], balsam fir [Abies balsamea], white cedar [Thuja occidentalis], and tamarack [Larix laricina]) in the forest overstory and was interspersed with white birch (Betula papyrifera) and quaking aspen (Populus tremuloides) due to logging and fires (Mech, 2009; see Heinselman, 1996 for a detailed description).

\section{Subjects, Materials, and Procedure}

As part of a long-term wolf radiocollaring study (Mech, 2009), our project captured wild gray wolves with modified foot-hold traps (Livestock Protection Company's EZ Grip 7) during May to November, 2011-2017 and 2019. Traps were buried just underground and generally located along logging roads and trails, oriented perpendicularly to the direction of travel, and checked at least daily. While there were no data on precise capture times of individual wolves, because traps were checked at least daily, capture time was always less than $24 \mathrm{hr}$. Further, because wolves tend to be crepuscular (Merrill \& Mech, 2003), and because the morning trap checks typically started at 0900, wolves were most likely captured either the night before or the morning of the daily checks (thus, wolves were likely captured for much less than the maximum 24 hours possible). The traps were baited with standard natural and commercial baits and lures. Trap set arrangements (blocking vegetation, branches/logs, small rocks, etc.) were designed to limit the space for final foot placement before the trap sprung to that of a single foot when a wolf closely approached the trap to investigate the bait (Figure 1). From this final foot placement, wolves would extend their head to visually and olfactorily investigate the bait (Figure 1) that was generally placed in a small dirt hole about 2.5-5 cm (1-2 in) in diameter and about 10-15 cm (4-6 in) deep, positioned about 28-30 cm (1112 in) behind the center of the trap. This arrangement resulted in a step test analog where body positioning was similar to that in a paw preference test when stepping across a gap to a platform (Aggestam \& Cahusac, 2007) or when taking a first step down (Tomkins et al., 2010) or dominant limb use for postural support (Wells et al., 2016). As part of processing captured wolves, the captured foot (front or back and left or right) was recorded, and sex and age class (pup or non-pup) were determined. 
Figure 1

Camera Trap Photos of a Gray Wolf(Canis lupus) Approaching and Investigating a Baited "Mock” Foot-Hold Trap Set

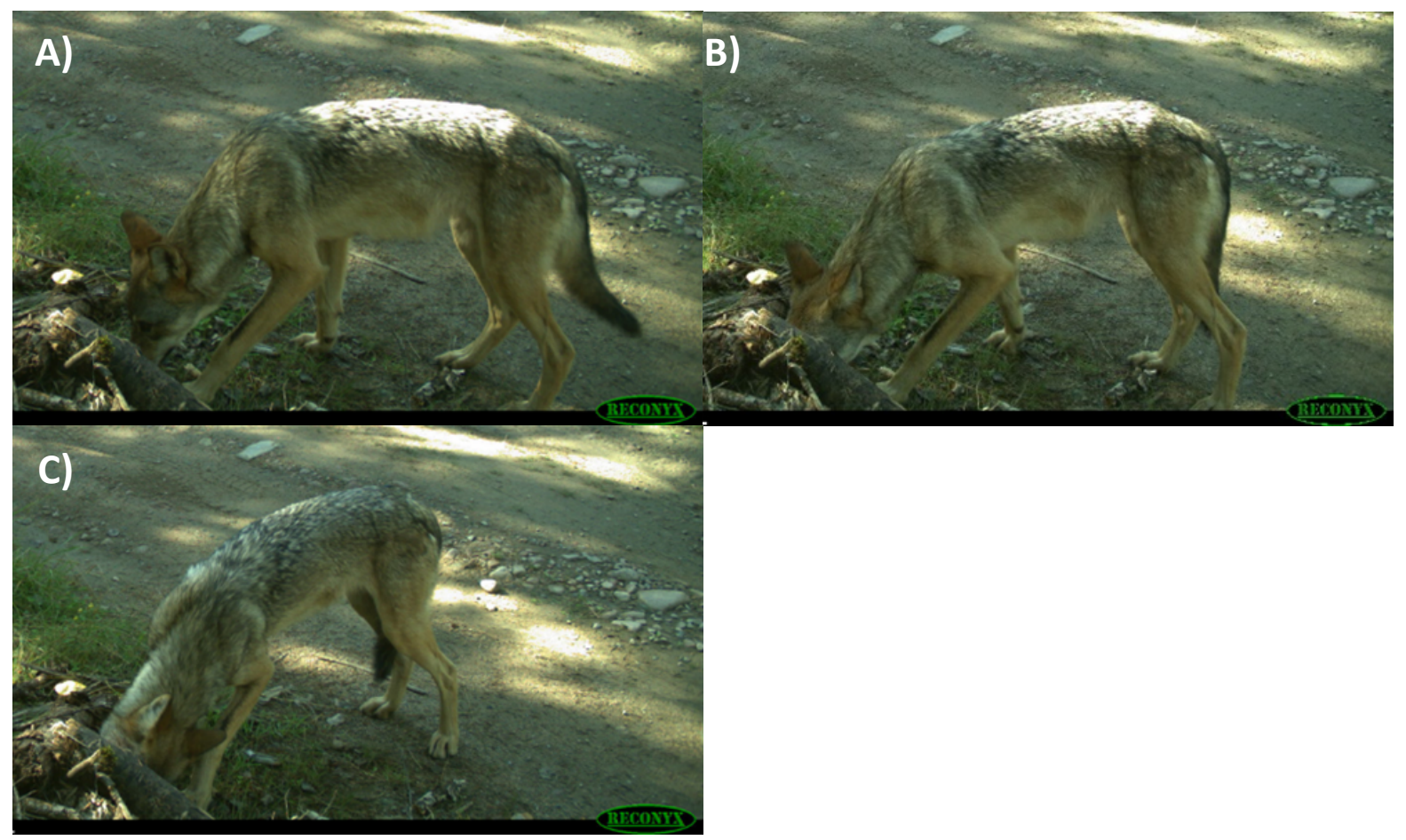

Note. No trap was present underground, but the above ground blocking/vegetation arrangement and baiting were done as usual when finishing a buried foot-hold trap set. Pictures were taken in the Superior National Forest, Minnesota, USA during summer 2015. Had there been a buried trap, the wolf's front left foot would have been captured (a step test analog). The wolf's progression is displayed in Figure 1A, 1B, and 1C. Images by S. Barber-Meyer.

\section{Analysis}

Lateralization was scored by which front foot was captured by the foot-hold trap (left or right). Only captures of a single front foot were analyzed. To avoid possible pseudoreplication and trap-response issues, recaptures of individual wolves were excluded. Thus, this study analyzed population-level lateralization (not individual-level) (Halpern et al., 2005). A $\chi^{2}$ goodness of fit test was conducted to determine if the observed proportion of capture by a left or right foot (i.e., lateralization) was different from chance (50:50). Logistic regression was used to examine whether sex or age class were predictors of which front foot was caught (with right $=0$ and left $=1$ in the model). Statistical tests were conducted in Statistix (version 10.0, Build: 1/29/2019, Analytical Software (C) 1985-2013) and considered significant at $\alpha=.05$.

\section{Results}

A total of 93 capture events were analyzed across the eight years comprising 49 females and 44 males, 79 non-pups and 14 pups, and 45 left and 48 right foot captures (Table 1). Eleven recaptures from 10 wolves that were captured and recaptured during this study period were excluded. No support was found for lateralization of the foot captured in wild wolves $\left(\chi^{2}=0.097, p=.756\right)$. Similarly, sex and age class were not significant predictors of which foot was captured (sex coefficient, $p=.857$; age class coefficient, $p=.471$; model deviance $=128.29, p=.005$; overall proportion of event outcomes correctly classified by model $=0.538$ ). 
Table 1

Counts of the Foot Captured by Sex and Age class of Wild Gray Wolves (Canis lupus)

\begin{tabular}{lcc}
\hline \multicolumn{1}{c}{ Sex } & Non-Pup & Pup \\
\hline Male & $17 \mathrm{~L}: 19 \mathrm{R}$ & $4 \mathrm{~L}: 4 \mathrm{R}$ \\
Female & $20 \mathrm{~L}: 23 \mathrm{R}$ & $4 \mathrm{~L}: 2 \mathrm{R}$ \\
\hline
\end{tabular}

Note. $\mathrm{L}=$ front left $: \mathrm{R}$ = front right. Data were collected in the Superior National Forest, Minnesota, USA from 2011-2017 and 2019.

\section{Discussion and Conclusions}

Contrary to my prediction, wild wolves did not exhibit lateralization in the front foot captured at the population level. Given inconsistent and / or inconclusive evidence of sex and age-related lateralization in dogs, it was not surprising that sex and age did not predict the foot that was captured in wolves. Either the wolf population sampled does not possess lateralization at the population level or information from a single foot-hold trapping event per wolf is not a reliable indicator. Trap sets were designed to limit final front foot placement to a single foot from where wolves would then extend their heads forward to investigate the bait (Figure 1) in a position similar to a step test. Because this study analyzed data from a step test analog that was apparently readily accomplished (based on the sample size), potential emotional difficulty of completing the task (e.g., frustration based on task complexity) was not, in this case, expected to influence the results (Wells, 2021). Thus, foot-hold trapping could serve as an indicator of lateralization if it exists in terms of the tendency to place weight on a particular front foot for postural support (the type of lateralization revealed when humans do a cartwheel, kick a ball, or ride a snowboard). However, if lateralization in wolves exists mainly in terms of paws used to manipulate an object (e.g., handedness in humans), foot-hold trapping results would not be a reliable indicator.

Although this analysis did not document population level lateralization in wild wolves, wild wolves may yet exhibit individual lateralization that would not be revealed by this step test analog where each wolf was only sampled once. Because many mammals exhibit lateralization (Reddon \& Hurd, 2009), it would be surprising if wolves do not possess lateralization at the individual level. Also, because lateralization is known to be task dependent (Halpern et al., 2005; Poyser et al., 2006; Tomkins et al., 2010), and because this analysis considered only a single task, lateralization (both population and individual) in wild wolves may yet be revealed by other tasks. Thus, additional research on wolf lateralization examining pawedness as revealed by multiple tasks, including the use of the paw to manipulate an object, could provide clarification. Because the method to calculate lateralization can also affect results (e.g., total touches, first touch, indices based on frequency of paw use or time spent using a paw, etc.) (Drew, 2015; Poyser et al., 2006), multiple analyses should be conducted to gain a better understanding of the potential degree and direction of any lateralization. While an exploratory study has been conducted with a small sample of captive wolves on two tasks (Drew, 2015), additional research that accounts for confounding factors (such as different rearing conditions and methods, Brown et al., 2007) may help elucidate lateralization in wolves. Examining potential trade-offs between the costs and benefits of lateralization that wolves may incur, would be very interesting in terms of evolution and in comparison with dogs. Others note that obligatory quadrupeds (such as wolves) may fundamentally differ in such handedness from primates (Poyser et al., 2006). This difference may also warrant additional research into these highly social animals (Lampe et al., 2017; Mech et al., 2015; Range \& Virányi, 2015) that ambidextrously attack prey and detect attacks from other wolves, while also exhibiting enhanced neural capacity and social cohesion often observed with lateralization (Vallortigara \& Rogers, 2005). Moreover, because lateralization has been linked to emotional functioning and animal welfare (Leliveld et al., 2013; Rogers, 2010; Siniscalchi et al., 2021; Wells, 2021), such research including baseline lateralization data from wild wolves could also inform captive wolf 
management and conservation, including the captive breeding programs for endangered Mexican wolves (C. l. baileyi) and red wolves and other programs (e.g., educational facilities).

\section{Acknowledgements}

Funding was provided by the U.S. Geological Survey (USGS). I thank numerous USGS volunteer field technicians for collecting data. I thank Dr. Rolf Peterson (Michigan Technical University, retired) for his query that spurred my interest. I thank Kimberly Rivera, Dr. Matthew Cobb, and two anonymous reviewers for comments on an earlier draft. Any mention of trade, product, or firm names is for descriptive purposes only and does not imply endorsement by the U.S. Government.

Conflict of interest: The author declared that no conflicts of interest exist.

\section{References}

Aggestam, F., \& Cahusac, P. M. B. (2007). Behavioral lateralization of tactile performance in the rat. Physiology and Behavior, 91, 335-339.

Austin, N. P., \& Rogers, L. J. (2012). Limb preferences and lateralization of aggression, reactivity and vigilance in feral horses, Equus caballus. Animal Behaviour, 83(1), 239-247.

Austin, N. P., \& Rogers, L. J. (2014). Lateralization of agonistic and vigilance responses in Przewalski horses (Equus przewalskii). Applied Animal Behaviour Science, 151, 43-50.

Barber-Meyer, S. M., \& Mech, L. D. (2014). How hot is too hot? Live-trapped gray wolf rectal temperatures and 1year survival. Wildlife Society Bulletin, 38, 767-772.

Batt, L., Batt, M., Baguley, J., \& McGreevy, P. (2008). Stability of motor lateralisation in maturing dogs. Laterality, $5,468-479$.

Berta, C. (2011). Lateralized behavior in domesticated dogs. ESSAI, 8, Article 9. http://dc.cod.edu/essai/vol8/iss1/9

Bisazza, A., Cantalupo, C., Capocchiano, M., \& Vallortigara, G. (2000). Population lateralization and social behavior: A study with sixteen species of fish. Laterality, 5, 269-284.

Björnerfeldt, S. (2007). Consequences of the domestication of man's best friend, the dog [Doctoral dissertation, Uppsala Universitet]. Digital comprehensive summaries of Uppsala dissertations from the Faculty of Science and Technology 289.

Bonati, B., Csermely, D., \& Romani, R. (2008). Lateralization in the predatory behavior of the common wall lizard (Podarcis muralis). Behavioural Processes, 79, 171-174.

Branson, N. J., \& Rogers, L. J. (2006). Relationship between paw preference strength and noise phobia in Canis familiaris. Journal of Comparative Psychology 120, 176-183.

Brown, C., Western, J., \& Braithwaite, V. (2007). The influence of early experience on, and inheritance of, cerebral lateralization. Animal Behavior, 74, 231-238.

Drew, L. (2015). Comparisons of laterality between wolves and domesticated dogs (Canis lupus familiarias) [Unpublished thesis]. Purchase College, State University of New York.

Fabre-Thorpe, M., Fagot, J., Lorincz, E., Levesque, F., \& Vauclair, J. (1993). Laterality in cats: Paw preference and performance in a visuomotor activity. Cortex, 29(1), 15-24.

Halpern, M. E., Güntürkün, O., Hopkins, W. D., \& Rogers, L. J. (2005). Lateralization of the vertebrate brain: Taking the side of model systems. The Journal of Neuroscience, 25, 10351-10357.

Heinselman, M. L. (1996). The boundary waters wilderness ecosystem. University of Minnesota Press.

Lampe, M., Bräuer, J., Kaminski, J., \& Virányi, Z. (2017). The effects of domestication and ontogeny on cognition in dogs and wolves. Scientific Reports, 7(1), 1-8.

Leliveld, L. M. C., Langbein, J., \& Puppe, B. (2013). The emergence of emotional lateralization: Evidence in nonhuman vertebrates and implications for farm animals. Applied Animal Behaviour Science, 145(1-2), 1-14.

MacNeilage, P. F., Studdert-Kennedy, M. G., \& Lindblom, B. (1987). Primate handedness reconsidered. Behavioral and Brain Sciences, 10(2), 247-263.

Maynard Smith, J. (1982). Evolution and the theory of games. Cambridge University Press.

McGrew, W. C., \& Marchant, L. F. (1999). Laterality of hand use pays off in foraging success for wild chimpanzees. Primates, 40(3), 509-513. 
Mech, L. D. (2009). Long-term research on wolves in the Superior National Forest. In A. P. Wydeven, T. R. VanDeelen, \& E. J. Heske (Eds.), Recovery of gray wolf in the Great Lakes Region of the United States: An endangered species success story (pp. 15-34). Springer.

Mech, L. D., Smith, D. W., \& MacNulty, D. R. (2015). Wolves on the hunt: The behavior of wolves hunting wild prey. University of Chicago Press.

Merrill, S. B., \& Mech, L. D. (2003). The usefulness of GPS telemetry to study wolf circadian and social activity. Wildlife Society Bulletin, 31(4), 947-960.

Nelson, M. E., \& Mech, L. D. (1981). Deer social organization and wolf depredation in northeastern Minnesota. Wildlife Monographs, 77, 3-53.

Neveu, P., \& Merlot, E. (2003). Cytokine stress responses depend on lateralization in mice. The International Journal on the Biology of Stress, 6, 5-9.

Poyser, F., Caldwell, C., \& Cobb, M. (2006). Dog paw preference shows lability and sex differences. Behavioural Processes, 73, 216-221.

Quaranta, A., Siniscalchi, M., Frate, A., \& Vallortigara, G. (2004). Paw preference in dogs: Relations between lateralised behaviour and immunity. Behavioural Brain Research, 153(2), 521-525.

Quaranta, A., Siniscalchi, M., \& Vallortigara, G. (2007). Asymmetric tail-wagging responses by dogs to different emotive stimuli. Current Biology, 17, R199-R201.

Range, F., \& Virányi, Z. (2015). Tracking the evolutionary origins of dog-human cooperation: The "Canine Cooperation Hypothesis". Frontiers in Psychology, 5, 1582, 1-10.

Reddon, A. R., \& Hurd, P. (2008). Aggression, sex and individual differences in cerebral lateralization in a cichlid fish. Biology Letters, 4, 338-340.

Reddon, A. R., \& Hurd, P. (2009). Acting unilaterally: Why do animals with strongly lateralized brains behave differently than those with weakly lateralized brains? Bioscience Hypotheses, 2, 383-387.

Rogers, L. J. (1989). Laterality in animals. International Journal of Comparative Psychology, 3, 5e25.

Rogers, L. J. (2010). Relevance of brain and behavioural lateralization to animal welfare. Applied Animal Behaviour Science, 127(1-2), 1-11.

Sikes, R. S., the Animal Care and Use Committee of the American Society of Mammalogists. (2016). Guidelines of the American Society of Mammalogists for the use of wild mammals in research. Journal of Mammalogy, 97, 663-688.

Siniscalchi, M., Bertino, D., d'Ingeo, S., \& Quaranta, A. (2019). Relationship between motor laterality and aggressive behavior in sheepdogs. Symmetry, 11(2), 233.

Siniscalchi, M., d'Ingeo, S., \& Quaranta, A. (2021). Lateralized emotional functioning in domestic animals. Applied Animal Behaviour Science, 237, 105282.

Tan, Ü. (1987). Paw preferences in dogs. International Journal of Neuroscience, 32, 825-829.

Tomkins, L. M., Thomson, P. C., \& McGreevy, P. (2010). First-stepping test as a measure of motor laterality in dogs (Canis familiaris). Journal of Veterinary Behaviour, 5, 247-255.

Vallortigara, G., Cozzutti, C., Tommasi, L., \& Rogers, L. J. (2001). How birds use their eyes: Opposite left-right specialisation for the lateral and frontal visual hemifield in the domestic chick. Current Biology, 11, 29-33.

Vallortigara, G., \& Rogers, L. J. (2005). Survival with an asymmetrical brain: Advantages and disadvantages of cerebral lateralization. Behavioral and Brain Sciences, 28, 575-589.

Versace, E., Morgante, M., Pulina, G., \& Vallortigara, G. (2007). Behavioural lateralization in sheep (Ovis aries). Behavioural Brain Research, 184, 72-80.

Wells, D. L. (2003). Lateralised behavior in the domestic dog, Canis familiaris. Behavioural Processes, 61, $27-35$.

Wells, D. L. (2021). Paw preference as a tool for assessing emotional functioning and welfare in domestic dogs and cats: A review. Applied Animal Behaviour Science, 236, 105148.

Wells, D. L., Hepper, P. G., Milligan, A. D., \& Barnard, S. (2016). Comparing lateral bias in dogs and humans using the Kong ${ }^{\mathrm{TM}}$ ball test. Applied Animal Behaviour Science, 176, 70-76.

Yoccoz, N. G. (1991). Use, overuse, and misuse of significance tests in evolutionary biology and ecology. Bulletin of the Ecological Society of America, 72(2), 106-111. 